\title{
Uso degli inibitori di pompa protonica in un Policlinico universitario
}

\author{
Proton pump inhibitor use in a university teaching hospital
}

\section{Maria Meli* ${ }^{*}$ Renato Malta, Luigi Aprea, Andrea Pasquale, Concetta La Seta, Salvatore Di Rosa, Natale D'Alessandro}

UO Farmacologia Clinica, Dipartimento Assistenziale dei Servizi Centrali d'Ospedale, Azienda Ospedaliera Universitaria Policlinico P. Giaccone, Palermo

Ricevuto il 28 marzo 2012; accettato il 15 giugno 2012

disponibile online il 28 luglio 2012

\section{KEY WORDS}

Proton pump inhibitors;

Drug utilization;

Prescribing

appropriateness;

Hospital;

DDD.

\begin{abstract}
Summary
Introduction: Proton pump inhibitors (PPIs) are highly prescribed drugs in Italy and in particular in the Sicilian region but little is known about their use in the hospital setting.

Materials and methods: PPI utilization and related costs were reviewed retrospectively by examining the pharmaceutical records of drug dispensation to the various wards of the Policlinico Universitario P. Giaccone of Palermo in 2010. Differences in the prescribing rates and drug preferences among the different clinical wards were analyzed.

Results: A total of 20,420 patients were hospitalized at the Policlinico of Palermo in 2010. Overall, the consumption of PPIs was 120 DDD/100 bed-days for the year 2010 with a total cost of 42,780 euros. Omeprazole and esomeprazole were the most commonly prescribed molecules accounting for over $70 \%$ of all prescriptions: nevertheless, wide differences in drug choices were noted even within the same ward. As expected, greater utilization rates were registered in the Internal Medicine and General Surgery departments. In particular, the highest consumption was observed in the Oncology, Geriatry and Obesity Surgery wards, with about 250 DDD/100 bed-days. All wards reported intravenous PPI administration suggesting some inappropriate use.

Discussion: From our data, PPIs appear to be moderately over-used at the Policlinico of Palermo. This practice may lead to the inappropriate continuation of therapy in primary care, further increasing costs and risks of adverse events. A survey evaluating in more detail the appropriateness of prescriptions is advisable.

(c) 2012 Elsevier Srl. All rights reserved.
\end{abstract}

* Corrispondenza: UO Farmacologia Clinica, Dipartimento dei Servizi Centrali d’Ospedale, Azienda Ospedaliera Universitaria Policlinico P. Giaccone, via del Vespro 129 - 90133 Palermo.

E-mail: maria.meli@unipa.it (M. Meli). 


\section{Introduzione}

Gli inibitori di pompa protonica (IPP) sono i farmaci più efficaci e utilizzati per ridurre la secrezione acida dello stomaco e si calcola che nel mondo la spesa annua per il loro impiego abbia superato i 25 miliardi di dollari [1]. In Italia, relativamente ai consumi territoriali, nel 2010 sono stati spesi 981 milioni di euro per gli IPP, che hanno rappresentato, così, la maggiore voce di spesa nell'ambito dei farmaci dell'apparato gastrointestinale e la seconda voce di spesa più alta globalmente dopo le statine [2]. In particolare, l'osservazione dei dati sul consumo degli IPP nel tempo, dal 2002 a oggi, ha mostrato un loro incremento progressivo; nel 2010 essi si sono attestati intorno alle 58 Defined Daily Doses (DDD)/1.000 abitanti/die con una prevalenza d'uso del 19\%. Inoltre, considerando l'insieme dei farmaci del sottogruppo ATC A02 (antiacidi, antimeteorici e antiulcera peptica), di cui gli IPP rappresentano la voce principale, il rapporto OsMed per l'anno 2010 [2] ha messo in luce come, tra le varie regioni italiane, sia la Sicilia ad avere il primato del maggiore consumo, che è stato di circa 104 DDD/1.000 abitanti/die a fronte di un valore medio nazionale di circa 64. Appare senz'altro utile, pertanto, avviare un'indagine approfondita che chiarisca le motivazioni di tale difformità nei consumi, in modo da accertare se possa essere attribuita a differenze inerenti alla popolazione trattata o a un diverso costume della classe medica, anche in relazione a un'inappropriatezza prescrittiva teoricamente correggibile. Inoltre, è da notare che poche informazioni sono disponibili riguardo ai consumi ospedalieri di queste molecole, a livello sia nazionale sia internazionale, mentre in ambito nosocomiale tale categoria di farmaci va incontro a un uso sempre più ampio, considerata la necessità di gestire pazienti più gravi o con più frequente alto rischio di ulcera peptica rispetto alla popolazione generale [3]. In effetti, un recente studio condotto presso l'Ospedale Sacco di Milano ha indicato che fino al $68 \%$ dei pazienti ricoverati assumeva IPP in modo inappropriato [4].

Si consideri che gli impieghi degli IPP attualmente approvati e riconosciuti dall'Agenzia Italiana del Farmaco (AIFA) ai fini della rimborsabilità, con due apposite note (1 e 48) [5,6], riguardano la terapia dell'ulcera peptica e della malattia da reflusso gastroesofageo, l'eradicazione dell'infezione da Helicobacter pylori e la prevenzione dell'ulcera da stress o indotta da trattamento cronico con farmaci antinfiammatori non steroidei o basse dosi di acido acetilsalicilico in pazienti a rischio (anziani o con ulcera pregressa), mentre altre loro possibili indicazioni, quali la semplice dispepsia, l'esofago di Barrett e le varici esofagee, rimangono controverse. Oltretutto, nel nostro Paese l'uso ospedaliero di queste molecole potrebbe non essere rigidamente aderente alle note AIFA, in quanto la prescrizione da parte del curante potrebbe presumibilmente avvenire anche sulla base di altre indicazioni, comunque supportate da dati di letteratura [7-9]. Di fatto, la prescrizione di IPP in assenza di una chiara indicazione è stata riportata in molti Paesi, sia a livello ospedaliero sia nell'uso territoriale, con percentuali che variano dal 40 all'81\% [1]. Ciò può comportare un incremento ingiustificato dei costi sanitari, oltre che degli effetti avversi prevenibili. In particolare, il trattamento cronico con IPP è stato associato a una maggiore frequenza di fratture osteoporotiche dell'anca, colite pseudomembranosa e infezioni respiratorie come la polmonite [10-12]. Inoltre, è nota la capacità degli IPP di interagire con gli enzimi coinvolti nella biotrasformazione di farmaci e xenobiotici e, quindi, di dar luogo a possibili interazioni con inconvenienti non sempre prevedibili [13-15].

Sulla base di queste premesse, è stata condotta un'indagine di farmacoutilizzazione relativa all'uso degli IPP presso il Policlinico Universitario di Palermo (AOUP P. Giaccone) nell'anno 2010. Quest'analisi dei consumi relativi alle diverse Unità Operative (UO) e ai loro corrispondenti

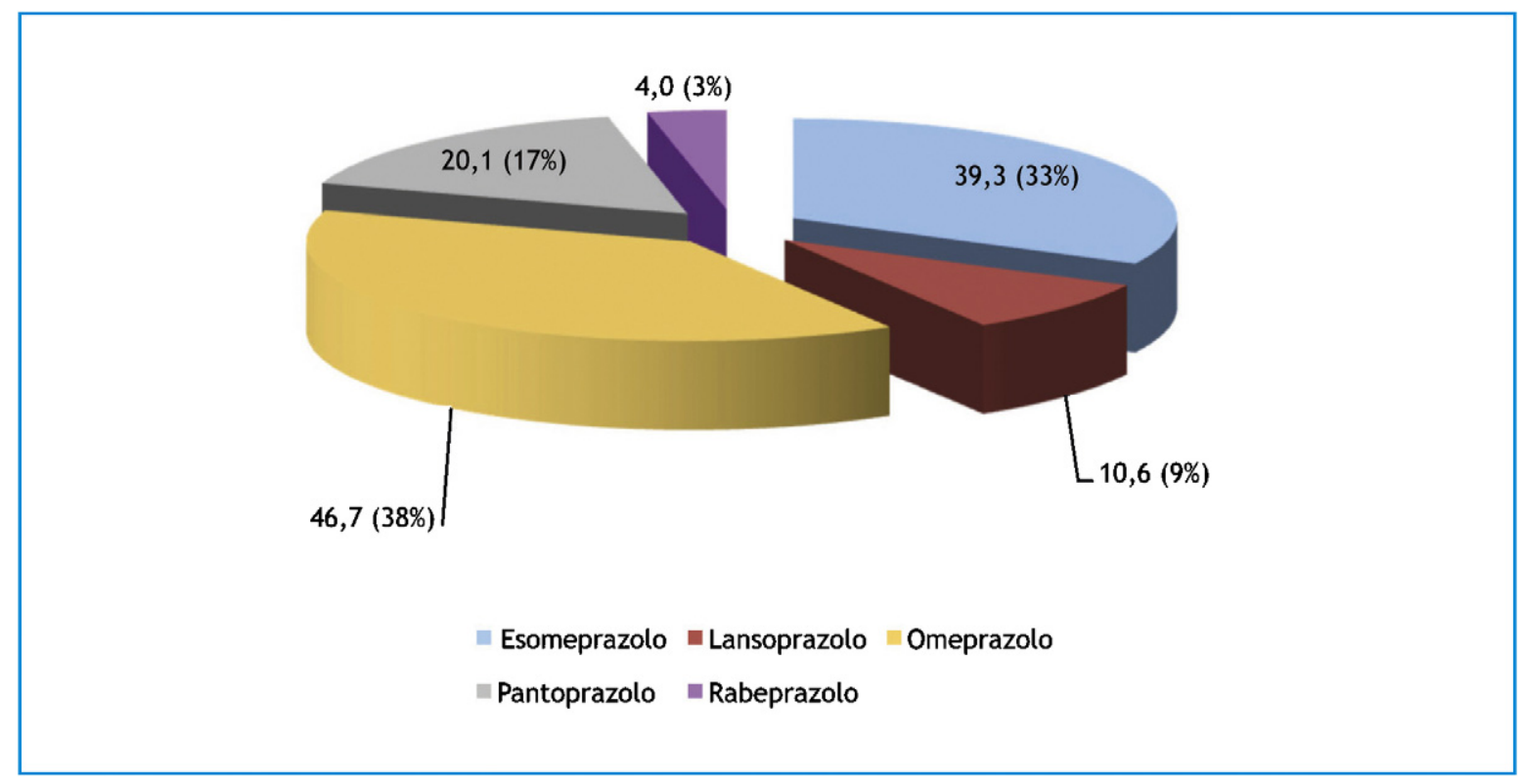

Figura 1 Consumo (in DDD/100 giorni di degenza e in percentuale sul consumo complessivo di IPP) delle diverse molecole di IPP presso l'Azienda Ospedaliera Universitaria Policlinico P. Giaccone nel 2010. 
ambiti disciplinari fornisce qualche suggerimento riguardo alle possibili inappropriatezze d'uso e costituisce la base di futuri studi mirati, fondati sull'esame delle singole prescrizioni e delle cartelle cliniche. I dati ottenuti potranno, inoltre, essere confrontati con quelli che saranno raccolti in tempi successivi, anche al fine di riscontrare gli effetti di possibili interventi messi in atto.

\section{Materiali e metodi}

L'indagine è stata condotta utilizzando la banca dati informatizzata della Farmacia Ospedaliera dell'AOUP P. Giaccone, ove vengono registrati tutti i farmaci dispensati ai reparti per la somministrazione ai pazienti in regime di ricovero ordinario (RO) o in day hospital (DH) e anche i farmaci che vengono consegnati ai pazienti per la prosecuzione della terapia al domicilio. Si tenga presente che nel nosocomio considerato, la prescrizione degli IPP avrebbe dovuto essere ancorata, secondo il Prontuario Terapeutico Ospedaliero Regionale, alle note AIFA, scegliendo i prodotti con un costo per DDD uguale o inferiore al valore di riferimento regionale e/o comunque con prezzo uguale o inferiore al generico corrispondente [16]. Di fatto, tutte e cinque le molecole erano disponibili, a prezzo ridotto, in quanto aggiudicatarie delle relative gare d'appalto per le specialità medicinali.

Il consumo dei farmaci è stato calcolato utilizzando il numero di DDD per 100 giorni di degenza e anche per numero di ricoveri, che costituiscono i parametri internazionalmente adottati per questo tipo di analisi; la DDD corrisponde alla dose media giornaliera per l'indicazione principale di ogni

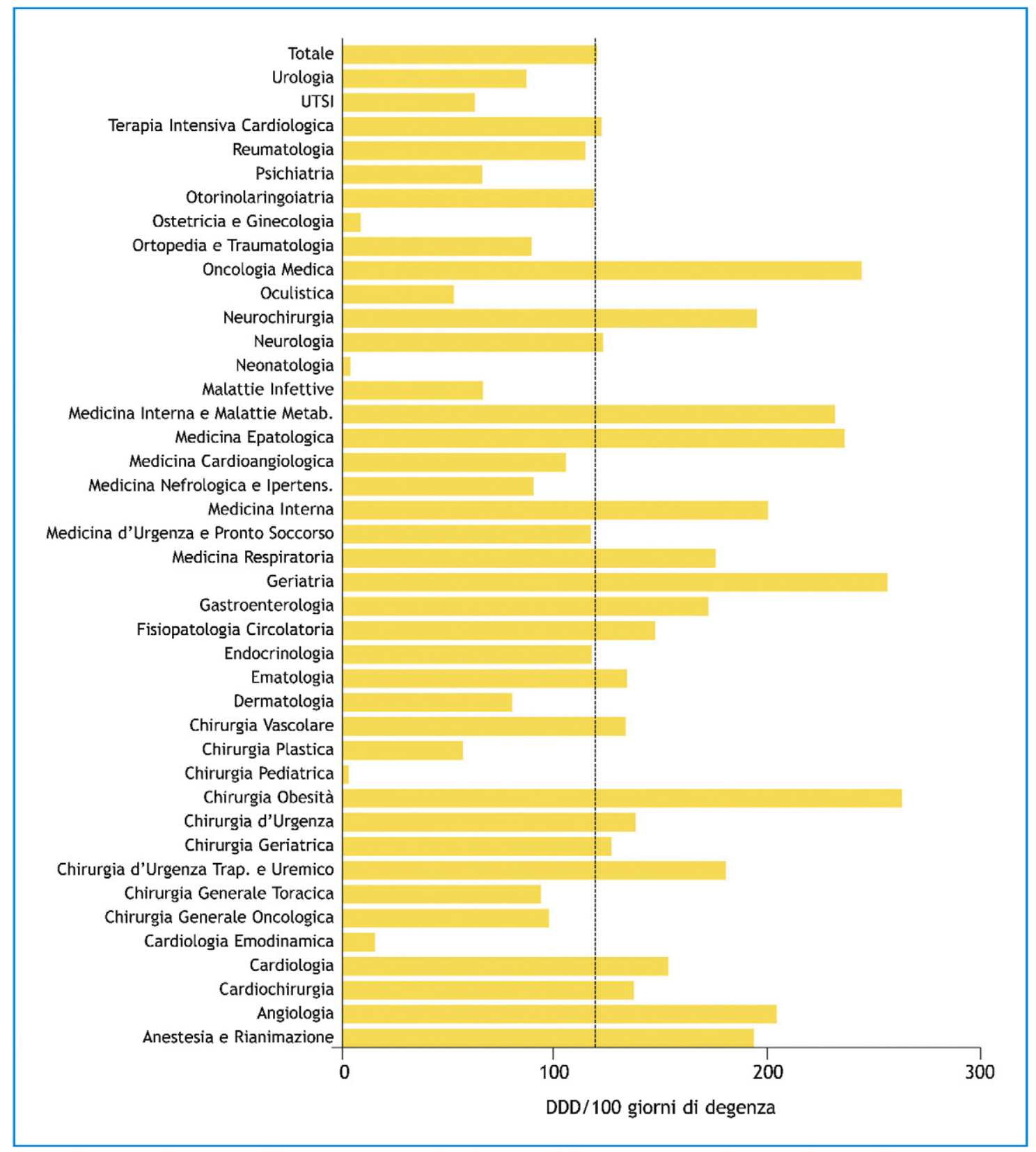

Figura 2 Consumo di IPP (in DDD/100 giorni di degenza) nelle diverse Unità Operative dell’Azienda Ospedaliera Universitaria Policlinico P. Giaccone (la linea tratteggiata indica il consumo totale). 
determinato farmaco nell'adulto. Il numero di DDD per 100 giorni di degenza è stato calcolato con la seguente formula:

(Numero DDD della confezione $) \times($ Numero di pezzi prescritti) $\times 100$

Numero di giorni di degenza - Numero di ricoveri

I valori di DDD impiegati per le varie molecole sono quelli aggiornati al 2009 dal WHO Collaborating Centre di Oslo. I risultati sono stati calcolati come valori complessivi riferiti all'intero ospedale (comprendente 508 posti letto di RO e 74 di $\mathrm{DH}$ ) o come valori disaggregati per ognuna delle $40 \mathrm{UO}$ che hanno fatto uso di queste molecole nell'anno 2010. La classificazione in macroreparti ospedalieri è stata effettuata secondo lo schema elaborato dal IMS Health, modificato dalla Società Italiana di Farmacia Ospedaliera e dei Servizi Farmaceutici alle Aziende Sanitarie (SIFO) [17].

\section{Risultati}

Nel 2010 sono stati ricoverati presso l'AOUP P. Giaccone 20.420 pazienti, per un numero complessivo di 131.446 giorni di degenza. Nello stesso periodo, sono state dispensate 155.797 DDD di IPP, per una spesa complessiva di 42.780 euro e una media di circa 120 DDD per 100 giorni di degenza. Questo dato appare relativamente alto se confrontato con quello di un ospedale di grandezza simile come quello di Trento (704 posti letto), dove, per esempio, per l'anno 2010 è stato riportato un consumo di circa 101 DDD/100 giorni di degenza; consumi più elevati sono stati riportati anche in Trentino relativamente a ospedali più piccoli, essendo il range osservato per l'intera regione compreso tra 97 e 135 DDD/100 giorni di degenza [18].

Sono stati quindi disaggregati i dati dell'AOUP P. Giaccone sulla base di alcune variabili, come per esempio il tipo di molecola prescritta. L'esame della distribuzione del consumo per molecola ha evidenziato che tutte le cinque molecole disponibili sono state prescritte, con una prevalenza dell'omeprazolo, che è stato l'IPP più impiegato (38\%) seguito dall'esomeprazolo (33\%) (fig. 1).

Nella maggior parte dei casi gli IPP sono stati somministrati per via orale, mentre un $26 \%$ circa dei pazienti è stato trattato per via endovenosa.

Per quanto riguarda l'analisi dei consumi di IPP per UO, questa è riportata nella figura 2. Tali dati dovrebbero essere parzialmente corretti per quei reparti che somministrano frequentemente le terapie in regime di DH (come l'Oncologia Medica), in quanto possono essere falsati dal fatto di riferire tutto il consumo a un numero relativamente piccolo di RO; tuttavia, non è stato possibile ottenere i dati delle prescrizioni disaggregati per $\mathrm{RO}$ e $\mathrm{DH}$.

Posta questa precisazione, un alto consumo è stato riscontrato nelle UO di Chirurgia dell'Obesità, di Geriatria e di Oncologia (che si sono attestate intorno a 250 DDD/100 giorni di degenza), ma anche in alcune UO di Medicina Interna e di Chirurgia e in Anestesia e Rianimazione. I dati sono stati

Tabella 1 Consumo di inibitori di pompa protonica presso l’AOUP P. Giaccone di Palermo nel 2010 per macroreparto.

\begin{tabular}{|c|c|c|c|c|c|c|c|c|}
\hline Unità operative & $\begin{array}{r}\text { Ricoveri } \\
(\mathrm{N})\end{array}$ & DDD & $\begin{array}{r}\text { DDD/100 giorni } \\
\text { di degenza }\end{array}$ & Rank & DDD/ricovero & Rank & $\begin{array}{r}\% \text { su totale } \\
\text { DDD }\end{array}$ & Rank \\
\hline Oncologia & 88 & 811 & 245,0 & 1 & 9,21 & 8 & 0,5 & 15 \\
\hline Pneumologia & 369 & 6.181 & 176,2 & 2 & 16,75 & 1 & 3,5 & 8 \\
\hline Medicina & 3.467 & 43.896 & 151,7 & 3 & 12,66 & 3 & 25,1 & 1 \\
\hline Terapia Intensiva & 582 & 8.457 & 150,1 & 4 & 14,53 & 2 & 4,8 & 6 \\
\hline Cardiologia & 1.285 & 11.858 & 147,9 & 5 & 9,22 & 7 & 6,8 & 5 \\
\hline Ematologia & 551 & 6.799 & 134,3 & 6 & 12,33 & 4 & 3,9 & 7 \\
\hline Pronto Soccorso & 2.773 & 16.862 & 133,4 & 7 & 6,08 & 11 & 9,6 & 4 \\
\hline Neurologia & 548 & 5.772 & 123,2 & 8 & 10,53 & 5 & 3,3 & 9 \\
\hline Endocrinologia & 398 & 4.380 & 118,3 & 9 & 11,00 & 6 & 2,5 & 11 \\
\hline Chirurgia & 4.913 & 36.085 & 110,9 & 10 & 7,34 & 9 & 20,6 & 2 \\
\hline Ortopedia & 752 & 4.542 & 88,9 & 11 & 6,03 & 12 & 2,6 & 10 \\
\hline Dermatologia & 544 & 1.656 & 80,0 & 12 & 3,04 & 15 & 0,9 & 13 \\
\hline Malattie Infettive & 427 & 2.633 & 65,9 & 13 & 6,16 & 10 & 1,5 & 12 \\
\hline Psichiatria & 302 & 1.480 & 64,8 & 14 & 4,90 & 13 & 0,8 & 14 \\
\hline Oculistica & 239 & 175 & 52,2 & 15 & 0,73 & 14 & 0,1 & 17 \\
\hline Ostetricia & 1.709 & 492 & 8,0 & 16 & 0,28 & 16 & 0,3 & 16 \\
\hline Pediatria & 62 & 14 & 0,8 & 17 & 0,22 & 17 & 0,1 & 18 \\
\hline Pazienti non ospedalizzati ${ }^{*}$ & & 22.686 & & & & & 13,0 & 3 \\
\hline Totale & 19.009 & 17.4779 & 245,0 & & 9,19 & & 100,0 & \\
\hline
\end{tabular}

* La voce si riferisce per la maggior parte dei casi $(87 \%)$ a farmaci dispensati ai pazienti per il primo ciclo di terapia al domicilio. 


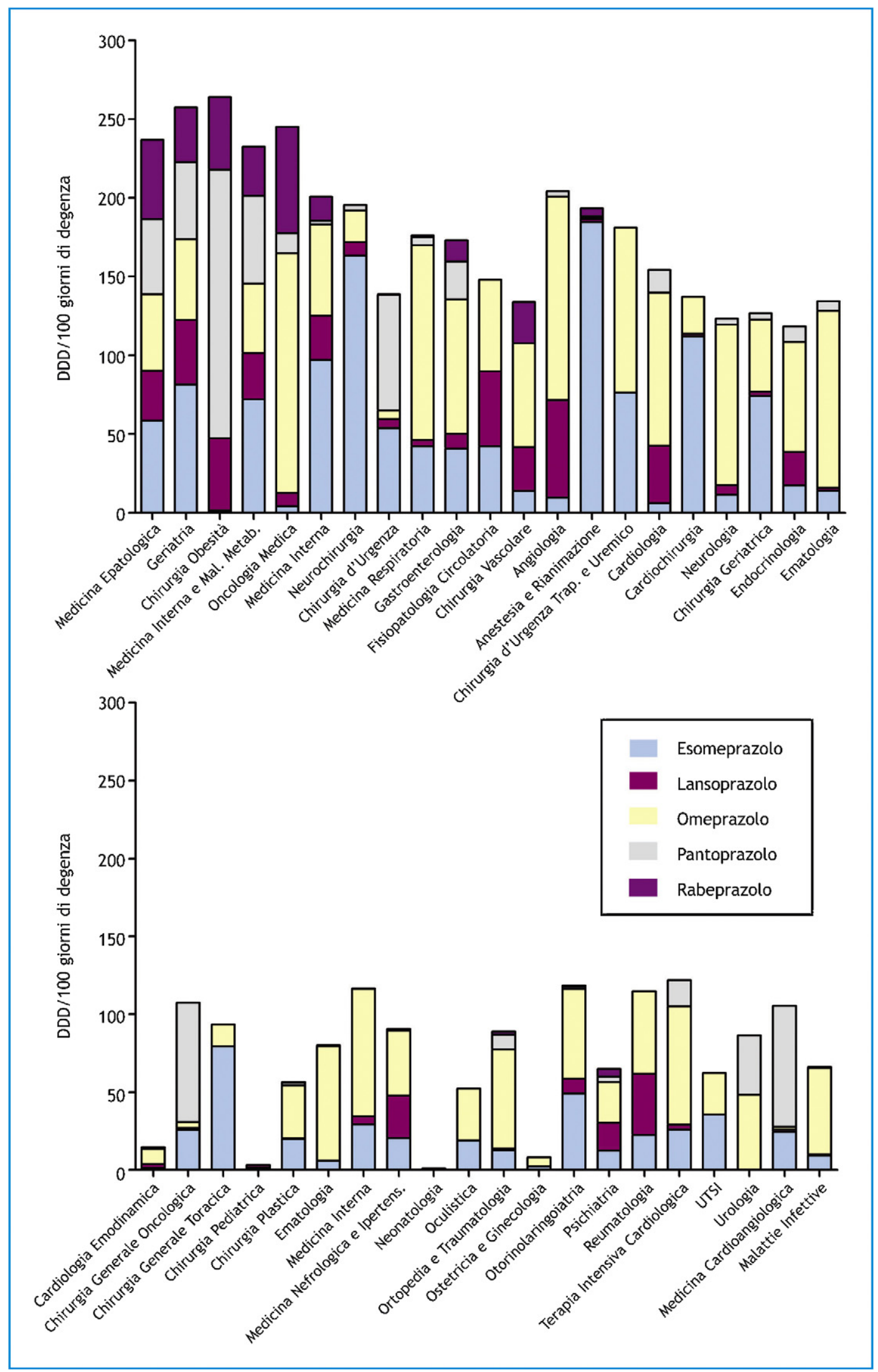

Figura 3 Consumo delle diverse molecole di IPP (in DDD/100 giorni di degenza) nelle Unità Operative dell'Azienda Ospedaliera Universitaria Policlinico P. Giaccone nell'anno 2010. 
anche analizzati in aggregato per macroreparto, unificando le UO che trattano patologie analoghe, in modo da avere un'idea del tipo d'uso che viene fatto nell'ospedale e, inoltre, per poter confrontare tali dati con quelli presenti in letteratura (tab. 1). Nella tabella sono indicate come voce a parte pure le DDD relative agli IPP che sono stati consegnati ai pazienti alla dimissione per la prosecuzione della terapia al domicilio e che sono state escluse dai dati di consumo in corso di ricovero.

Dall'osservazione della tabella, in accordo con $i$ dati nazionali riportati dalla SIFO [19], si rileva un maggiore impiego di IPP nelle UO di Medicina, Oncoematologia, Cardiologia e Terapia Intensiva: tuttavia, rispetto ai dati della SIFO, anche altri macroreparti come quelli di Neurologia e Pneumologia hanno mostrato consumi elevati.

Inoltre, è stato valutato il tipo di molecole impiegate nelle varie UO (fig. 3). Si noti la presenza di una notevole eterogeneità prescrittiva tra le 40 UO esaminate: infatti, solo il $15 \%$ circa di esse ha mostrato una preferenza specifica per una singola molecola, mentre circa il $30 \%$ ha utilizzato almeno tre diverse molecole.

Infine, dall'analisi effettuata, è emerso che pressoché tutte le UO hanno utilizzato gli IPP per via endovenosa, sebbene con una prevalenza in Anestesia e Rianimazione e in Chirurgia (fig. 4).

\section{Conclusioni}

Gli IPP sono tra i farmaci più utilizzati tanto in ambito territoriale quanto in ambito ospedaliero in tutto il mondo. La recente evidenza di possibili rischi relativi sia agli effetti avversi, tra cui quelli di tipo infettivologico, sia a interazioni con altri farmaci cosomministrati [13-15] ha indotto a riconsiderare criticamente il potenziale eccessivo consumo di tali molecole alla luce delle possibili conseguenze cliniche, oltre che farmacoeconomiche. In quest'ottica, da più parti è stata parimenti sottolineata la necessità di elaborare linee guida più esaustive, in modo da promuovere una maggiore appropriatezza prescrittiva da parte dei clinici [4]. D'altronde, occorre altresì considerare che, spesso, ciò che è "appropriato" (o forse meglio "adeguato") per il singolo paziente può discostarsi anche in modo significativo da quanto dettato da linee guida (si vada, per esempio, la necessità della semplificazione delle terapie nei pazienti anziani e portatori di più patologie).

Poiché la Sicilia è tra le regioni italiane con i più alti consumi, ci si è chiesti quale fosse il grado di farmacoutilizzazione a livello ospedaliero e sono stati, dunque, indagati preliminarmente i consumi di IPP in un ospedale universitario quale l'AOUP P. Giaccone di Palermo.

Dai dati esaminati, il consumo di queste molecole nell'AOUP di Palermo per l'anno 2010 è risultato relativamente alto se confrontato con i pochi resoconti nazionali a disposizione. Ciò appare in accordo con i dati regionali circa gli elevati consumi a livello territoriale, ma anche con quanto riportato dalla letteratura internazionale [20-22]. Sebbene il livello di spesa registrato possa apparire trascurabile, se confrontato con altre classi di farmaci, occorre considerare che la somministrazione ospedaliera è spesso seguita da una prescrizione ai pazienti alla dimissione e alla prosecuzione della terapia da parte dei medici di famiglia. In tale ottica, un ospedale universitario dovrebbe svolgere un ruolo di guida virtuosa nei confronti del territorio. Il fatto che tutte le

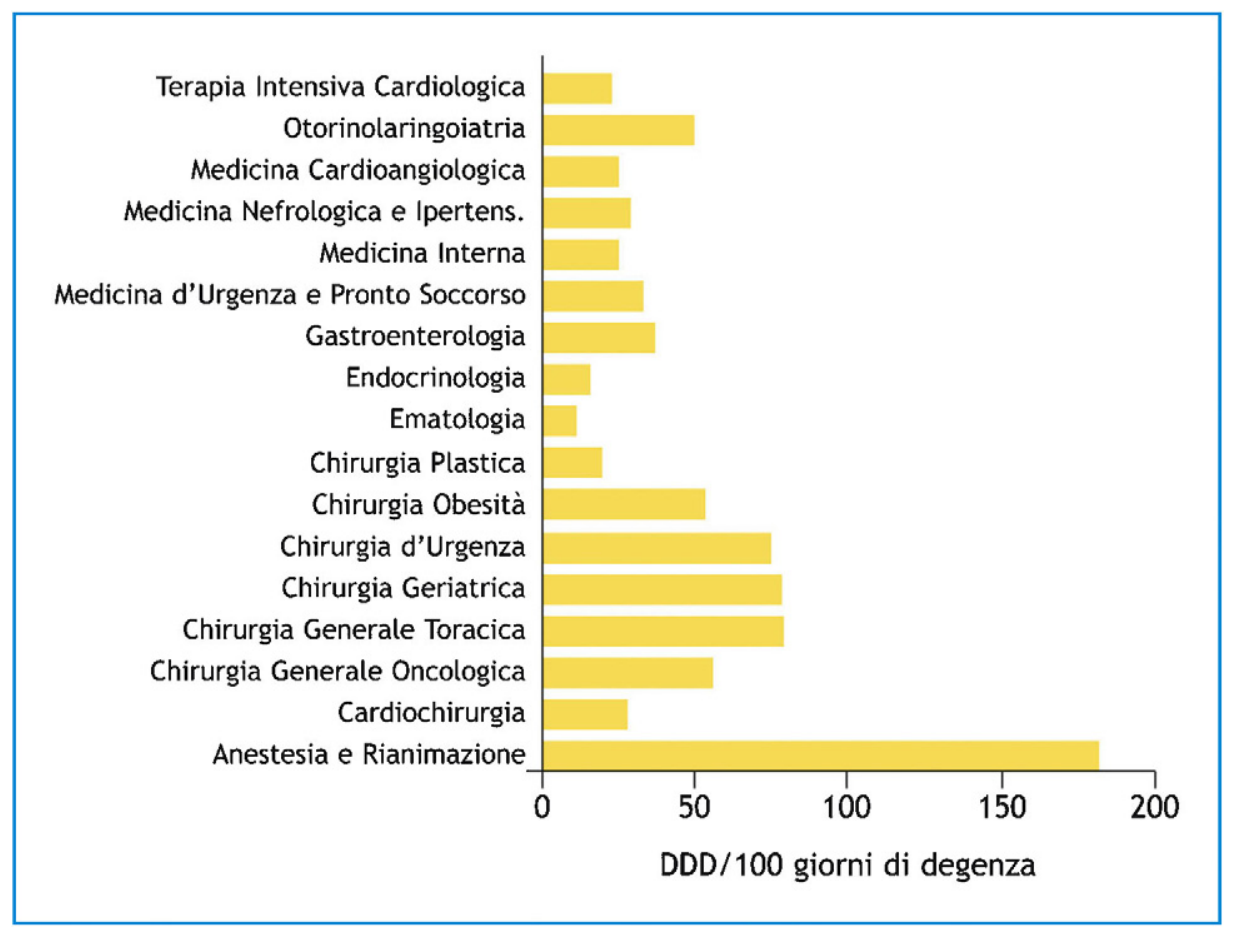

Figura 4 Consumo di IPP per via endovenosa (in DDD/100 giorni di degenza) presso l'Azienda Ospedaliera Universitaria Policlinico P. Giaccone nell'anno 2010: sono mostrate le Unità Operative che hanno dispensato più di 500 DDD per via endovenosa. 
cinque molecole disponibili siano state prescritte potrebbe apparire ingiustificato alla luce della loro supposta sostanziale equivalenza per quanto attiene al profilo farmacologico. In effetti, va detto che, nonostante sia stato evidenziato che esistono differenze tra $i$ vari IPP riguardo alla biodisponibilità, alla velocità di comparsa e durata dell'effetto, come pure alla capacità di causare determinate interazioni farmacologiche, mancano confronti diretti in letteratura a dosi equiattive [23-25].

Elevato è apparso altresì il consumo di IPP per via endovenosa, specie in relazione ai pazienti ricoverati in UO diverse dalla Terapia Intensiva, confermando la tendenza all'uso inappropriato di questa via di somministrazione in ambito ospedaliero riportata da altri autori $[26,27]$. Si ricordi che la somministrazione endovenosa comporta maggiori costi e rischi per il paziente. Attualmente essa è consigliabile nei pazienti con o $a$ rischio di sanguinamento gastrointestinale, nei pazienti con stati ipersecretori patologici (sindrome di Zollinger-Ellison), nei pazienti con impossibilità ad assumere farmaci per via orale e nella profilassi dell'ulcera da stress in pazienti critici [28,29].

Nel complesso, i risultati qui esposti non consentono di identificare con precisione le cause dell'aumentato consumo di IPP nell'AOUP P. Giaccone, anche se è facile ipotizzare che possa esservi stato un eccesso di prescrizioni in quelle UO nelle quali si è osservato un maggior uso. $\mathrm{Ci}$ si propone, dunque, di continuare l'indagine studiando in dettaglio l'appropriatezza prescrittiva al fine di poter suggerire eventuali interventi correttivi.

D'altra parte, occorre rilevare che in alcuni casi, per esempio in ambito oncoematologico, l'elevato consumo potrebbe essere stato causato dalla necessità di trattare condizioni cliniche per le quali, come già sottolineato, sebbene non esistano indicazioni specifiche in scheda tecnica, vi sono significativi dati nella letteratura internazionale che ne possono giustificare l'impiego nell'ambito di una sostanziale "good clinical practice", suggerendo ulteriormente che sarebbe auspicabile l'elaborazione di linee guida più accurate.

\section{Conflitto di interessi}

Gli autori dichiarano di non aver nessun conflitto di interessi.

\section{Bibliografia}

[1] Ahrens D, Chenot JF, Behrens G, Grimmsmann T, Kochen MM. Appropriateness of treatment recommendations for PPI in hospital discharge letters. Eur J Clin Pharmacol 2010;66(12): 1265-71.

[2] Gruppo di lavoro OsMed. L'uso dei farmaci in Italia. Rapporto nazionale anno 2010. Roma: Il Pensiero Scientifico; 2011.

[3] Gingold AR, Narasimhan G, Augello S, Clain DJ. The prevalence of proton pump inhibitor use in hospitalized patients. Pract Gastroenterol 2006;29:24-34.

[4] Parente F, Cucino C, Gallus S, Bargiggia S, Greco S, Pastore L, et al. Hospital use of acid-suppressive medications and its fallout on prescribing in general practice: a 1-month survey. Aliment Pharmacol Ther 2003;17(12):1503-6.
[5] Agenzia Italiana del Farmaco. Note AIFA 2009. Una guida per l'uso appropriato dei farmaci. Bollettino Informazione Farmaci 2009;16(5-6):193-7.

[6] Agenzia Italiana del Farmaco. Note AIFA 2009. Una guida per l'uso appropriato dei farmaci Bollettino Informazione Farmaci 2009;16(5-6):220-2.

[7] Sartori S, Trevisani L, Nielsen I, Tassinari D, Panzini I, Abbasciano V. Randomized trial of omeprazole or ranitidine versus placebo in the prevention of chemotherapy-induced gastroduodenal injury. J Clin Oncol 2000;18(3):463-7.

[8] Steer CB, Harper PG. Gastro-oesophageal complications in patients receiving cancer therapy: the role of proton pump inhibitors. Eur J Gastroenterol Hepatol 2002;14(Suppl. 1):S1721.

[9] Wang WH, Huang JQ, Zheng GF, Xia HH, Wong WM, Liu XG, et al. Effects of proton-pump inhibitors on functional dyspepsia: a meta-analysis of randomized placebo-controlled trials. Clin Gastroenterol Hepatol 2007;5(2):178-85.

[10] Loo VG, Bourgault AM, Poirier L, Lamothe F, Michaud S, Turgeon $\mathrm{N}$, et al. Host and pathogen factors for Clostridium difficile infection and colonization. N Engl J Med 2011;365(18):1693703.

[11] Yang YX, Lewis JD, Epstein S, Metz DC. Long-term proton pump inhibitor therapy and risk of hip fracture. JAMA 2006;296(24): 2947-53.

[12] Laheij RJ, Sturkenboom MC, Hassing RJ, Dieleman J, Stricker $\mathrm{BH}$, Jansen JB. Risk of community-acquired pneumonia and use of gastric acid-suppressive drugs. JAMA 2004;292(16):1955-60.

[13] Ogawa R, Echizen H. Drug-drug interaction profiles of proton pump inhibitors. Clin Pharmacokinet 2010;49(8):509-33.

[14] Yang YX, Metz DC. Safety of proton pump inhibitor exposure. Gastroenterology 2010;139(4):1115-27.

[15] Montanari P. Gastroprotezione con inibitori di pompa protonica in ospedale: oltre all'(ab)uso, anche eventi avversi? Ital J Med 2010;4(1):51-6.

[16] Prontuario Terapeutico Ospedaliero Territoriale della Regione Siciliana. Aggiornamento del 28 dicembre 2009. Suppl Ord GURS 22 gennaio 2010;3(Pt 1):4.

[17] Biasi V, Cassani T, Costa E, Scroccaro G. Precisazioni sulle classificazioni e i parametri utilizzati nelle elaborazioni. In: Società Italiana di Farmacia Ospedaliera e dei Servizi Farmaceutici delle Aziende Sanitarie. Report SIFO-IMS sulle prescrizioni ospedaliere 2008-2009. Verona: SIFO; 2009. p. 7-8.

[18] Spanti D, Zanetti G, Tibaldo C, Fonzi E, Pasqualini A, Permiani G, et al. L'utilizzo dei farmaci in ospedale. In: Azienda Provinciale per i Servizi Sanitari. L'uso dei farmaci in Trentino. Rapporto 2010. Trento: APSS; 2010. p. 58-76.

[19] Biasi V, Cassani T, Costa E, Scroccaro G. I macroreparti. In: Società Italiana di Farmacia Ospedaliera e dei Servizi Farmaceutici delle Aziende Sanitarie. Report SIFO-IMS sulle prescrizioni ospedaliere 2008-2009. Verona: SIFO; 2009. p. 30-80.

[20] Mat Saad AZ, Collins N, Lobo MM, O'Connor HJ. Proton pump inhibitors: a survey of prescribing in an Irish general hospital. Int J Clin Pract 2005;59(1):31-4.

[21] Niklasson A, Bajor A, Bergendal L, Simrén M, Strid H, Björnsson $\mathrm{E}$. Overuse of acid suppressive therapy in hospitalised patients with pulmonary diseases. Respir Med 2003;97(10): 1143-50.

[22] Strid H, Simrén $M$, Björnsson ES. Overuse of acid suppressant drugs in patients with chronic renal failure. Nephrol Dial Transplant 2003;18(3):570-5.

[23] Horn J. The proton-pump inhibitors: similarities and differences. Clin Ther 2000;22(3):266-80.

[24] Kromer W, Horbach S, Lühmann R. Relative efficacies of gastric proton pump inhibitors: their clinical and pharmacological basis. Pharmacology 1999;59(2):57-77. 
[25] Sachs G, Shin JM, Howden CW. Review article: the clinical pharmacology of proton pump inhibitors. Aliment Pharmacol Ther 2006;23(Suppl. 2):2-8.

[26] Nasser SC, Nassif JG, Dimassi HI. Clinical and cost impact of intravenous proton pump inhibitor use in non-ICU patients. World J Gastroenterol 2010;16(8):982-6.

[27] Craig DG, Thimappa R, Anand V, Sebastian S. Inappropriate utilization of intravenous proton pump inhibitors in hospital practice. A prospective study of the extent of the problem and predictive factors. QJM 2010;103(5):327-35.
[28] Guda NM, Noonan M, Kreiner MJ, Partington S, Vakil N. Use of intravenous proton pump inhibitors in community practice: an explanation for the shortage? Am J Gastroenterol 2004;99(7): 1233-7.

[29] Metz DC, Forsmark C, Lew EA, Starr JA, Soffer EF, Bochenek W, et al. Replacement of oral proton pump inhibitors with intravenous pantoprazole to effectively control gastric acid hypersecretion in patients with Zollinger-Ellison syndrome. Am J Gastroenterol 2001;96(12): 3274-80. 